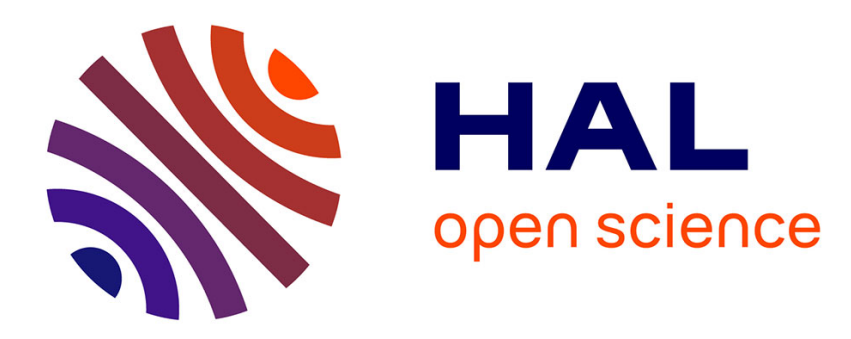

\title{
Adaptive Estimation Based on Quantized Measurements
}

Rodrigo Cabral Farias, Jean-Marc Brossier

\section{To cite this version:}

Rodrigo Cabral Farias, Jean-Marc Brossier. Adaptive Estimation Based on Quantized Measurements. ICC 2013 - IEEE International Conference on Communications, Jun 2013, Budapest, Hungary. pp.CT04/5. hal-00875981

\section{HAL Id: hal-00875981 https://hal.science/hal-00875981}

Submitted on 23 Oct 2013

HAL is a multi-disciplinary open access archive for the deposit and dissemination of scientific research documents, whether they are published or not. The documents may come from teaching and research institutions in France or abroad, or from public or private research centers.
L'archive ouverte pluridisciplinaire HAL, est destinée au dépôt et à la diffusion de documents scientifiques de niveau recherche, publiés ou non, émanant des établissements d'enseignement et de recherche français ou étrangers, des laboratoires publics ou privés. 


\title{
Adaptive Estimation Based on Quantized Measurements
}

\author{
Rodrigo Cabral Farias, and Jean-Marc Brossier \\ Gipsa-lab, Images and Signal Department \\ BP 46, 38402 Saint Martin d'Hères, France \\ Email: rodrigo.cabral-farias@gipsa-lab.grenoble-inp.fr, jean-marc.brossier@gipsa-lab.grenoble-inp.fr
}

\begin{abstract}
In this paper, the tracking of a slowly varying scalar Wiener process based on quantized noisy measurements is studied. An adaptive algorithm using a quantizer with adjustable input gain and bias is presented as a low complexity solution. The mean and asymptotic mean squared error of the algorithm are derived. Simulations under Cauchy and Gaussian noise are presented to validate the results and a comparison with the optimal estimator in the Gaussian and real-valued measurement case shows that the loss of performance due to quantization is negligible using 4 or 5 bits of resolution.
\end{abstract}

Index Terms-Adaptive estimation, quantization, tracking loops.

\section{INTRODUCTION}

With the evolution of sensor and communication technology, research on applications using sensor networks increased substantially. Sensor network applications can be found in a broad range of domains: environmental monitoring, agricultural monitoring, health care and many others [1]. Most of the research in this area is focused on the estimation part of sensing or in routing, while implementation constraints such as bandwidth and complexity are neglected.

Quantization of the measurements is an efficient way of taking into account bandwidth and complexity constraints. In quantization literature [2], most of the results are obtained for minimization of the measurements reconstruction error, while only a few results appear for the maximization of the estimation performance based on quantized measurements. The latter is clearly the main issue in sensing applications.

In [3], the performance of estimation of a constant parameter based on quantized noisy measurements is studied, it is shown that a good choice for the quantizer input offset is to use feedback from the output. It is also shown that for constant input offset, binary quantization and Gaussian noise, the best offset is exactly the parameter value and the best estimation asymptotic variance, given by the Cramér-Rao bound (CRB), is $\frac{\pi}{2}$ times the real-valued measurement CRB. Motivated by this small loss of performance, the estimation of a constant parameter based on binary quantized measurements with Gaussian noise was studied in [4]. An adaptive scheme for placing the threshold at the last estimate is proposed but not detailed. Sensor network schemes for adaptively replacing the thresholds are later developed and analyzed in [5] and [6]. In [5] a simple additive recursive algorithm is used for updating the thresholds at the sensors, while in a fusion center, the binary measurements are used to obtain a maximum likelihood estimate of the parameter. More complex threshold update schemes are presented in [6].

Some unsolved problems can be found in the references mentioned above. To reach the performance in [3], [5], [6] complex maximum likelihood algorithms must be applied. Also, one can be interested in tracking a slowly variable parameter instead of a constant. Thus, in this paper, a low complexity adaptive algorithm for tracking a slowly variable location parameter from noisy multiple bit measurements is proposed. The parameter is modeled by a Wiener process and the noise is considered to be symmetrically distributed. The estimation performance is analyzed using adaptive algorithms theory and after maximizing the performance with respect to (w.r.t.) its parameters, simulation results are presented under Gaussian and Cauchy noise.

\section{PRoblem StATEMENT AND ADAPTIVE ALGORITHM}

The stochastic process to be tracked is a scalar Wiener process given by the following model:

$$
X_{k}=X_{k-1}+W_{k},
$$

where $k \in \mathbb{N}^{\star}$ is the sample (time) index, $W_{k}$ is a sequence of independent and identically distributed (i.i.d.) zero mean Gaussian random variables (r.v.) with small standard deviation $W_{k} \sim \mathcal{N}\left(0, \sigma_{w}^{2}\right), \sigma_{w} \ll 1$. The initial condition $X_{0}$ is an unknown constant $x$.

The signal $X_{k}$ is measured with additive noise:

$$
Y_{k}=X_{k}+V_{k}
$$

where $Y_{k}$ is the measurement and the noise is $V_{k}$. The measurements are quantized using an adjustable quantizer with the following input-output relation:

$$
i_{k}=Q\left(\frac{Y_{k}-b_{k}}{\Delta_{k}}\right),
$$

where $i_{k} \in\left\{-\frac{N_{I}}{2}, \cdots,-1,+1, \cdots, \frac{N_{I}}{2}\right\}, N_{I}$ is the number of quantization intervals and $b_{k}$ and $\frac{1}{\Delta_{k}}$ are sequences of adjustable quantizer offsets and input gains respectively. The quantizer is parametrized also by its vector of thresholds defining the quantization intervals

$$
\boldsymbol{\tau}=\left[\begin{array}{lllllll}
\tau_{-\frac{N_{I}}{2}} & \cdots & \tau_{-1} & \tau_{0} & \tau_{1} & \cdots & \tau_{\frac{N_{I}}{2}}
\end{array}\right]
$$


Some assumptions will now be stated for simplification purposes.

- $V_{k}$ is assumed to be i.i.d. with a locally Lipschitz continuous cumulative distribution function (CDF) $F(x)$ and a probability density function (PDF) $f(x)$ that is an even function strictly decreasing w.r.t. $|x|$. The noise is parametrized by a known scale factor $\delta$, meaning that $F(x)=F_{n}\left(\frac{x}{\delta}\right)$, where $F_{n}$ is the CDF for $\delta=1$.

- The quantizer has symmetric thresholds:

$$
\begin{aligned}
& \tau_{0}=0, \quad \tau_{\frac{N_{I}}{2}}=+\infty, \\
& \tau_{i}=-\tau_{-i}, \quad \forall i \in\left\{1, \cdots, \frac{N_{I}}{2}\right\},
\end{aligned}
$$

thus its output is $i_{k}=i \operatorname{sign}\left(Y_{k}-b_{k}\right)$ for $\frac{\left|Y_{k}-b_{k}\right|}{\Delta_{k}} \in\left[\tau_{i-1}, \tau_{i}\right)$.

The objective is to track $X_{k}$ with an estimator $\hat{X}_{k}$ that uses the outputs of the adjustable quantizer. For doing so, the quantizer offset and input gain will be set to be

$$
b_{k}=\hat{X}_{k-1}, \quad \Delta_{k}=\Delta=c_{\delta} \delta,
$$

where $c_{\delta}$ is a free constant parameter that can be chosen to maximize estimation performance when the thresholds are fixed. With these parameters the amplitude information from the quantized measurements is enhanced. As the quantized measurement can be seen as a type of quantized innovation and as the signal to be estimated is a Wiener process, a low complexity choice for the estimator is a Kalman like algorithm with a constant gain:

$$
\hat{X}_{k}=\hat{X}_{k-1}+\gamma \eta\left[Q\left(\frac{Y_{k}-\hat{X}_{k-1}}{\Delta}\right)\right],
$$

$\gamma$ is a small positive constant gain, $\eta[i]=\eta_{i}$ is a sequence of $N_{I}$ coefficients $\left\{\eta_{-\frac{N_{I}}{2}} \ldots \eta_{\frac{N_{I}}{2}}\right\}$, they will be considered to be an odd function of $i$ and to be positive for positive $i$. Thus the positive coefficients are denoted in vector form as $\boldsymbol{\eta}=\left[\eta_{1} \ldots \eta_{\frac{N_{I}}{2}}\right]^{T}$.

Note that in the binary case $N_{I}=2$, the proposed method is similar to delta modulation. For $N_{I}>2$, the algorithm can be seem as a predictive quantizer intended for estimation of $X_{k}$ and not for reconstruction of $Y_{k}$.

\section{ESTIMATION PERFORMANCE}

The estimation algorithm (6) belongs to a wide class of adaptive algorithms studied in [7]. The tracking algorithm with the adjustable quantizer is depicted in Fig. 1. The results from [7, Ch. 4] can be applied to obtain its performance in terms of mean error and asymptotic mean squared error (MSE). The mean behavior of $\hat{X}_{k}$ can be approximated by the ordinary differential equation (ODE)

$$
\frac{d \hat{x}}{d t}=h(\hat{x}),
$$

where the correspondence between continuous and discrete time is $t_{k}=k \gamma$ and $h(\hat{x})$ is the following expectation w.r.t.

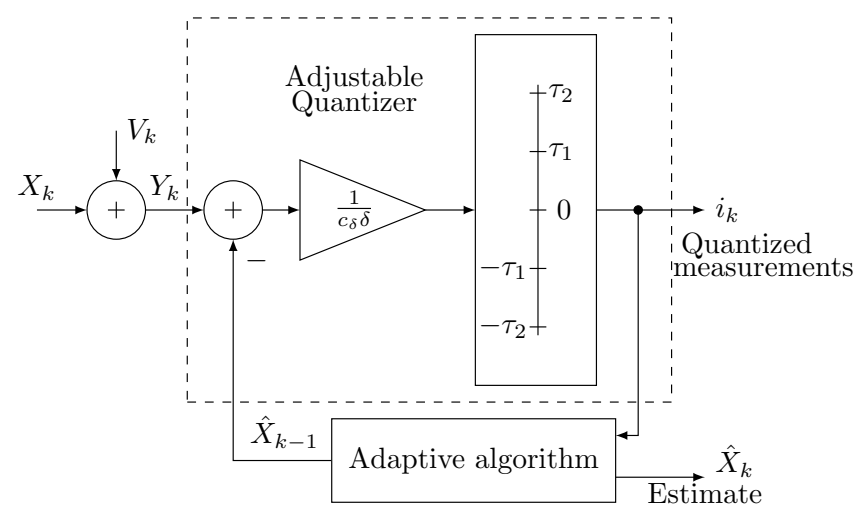

Fig. 1. Scheme representing the adjustable quantizer. The offset can be adjusted dynamically while the quantizer thresholds are fixed.

the probability measure of the noise r.v. $V$ :

$$
\begin{aligned}
h(\hat{x}) & =\mathbb{E}\left[\eta\left(Q\left(\frac{x-\hat{x}+V}{c_{\delta} \delta}\right)\right)\right] \\
& =\sum_{i=1}^{\frac{N_{I}}{2}} \eta_{i}\left[F_{d}(i, \hat{x}, x)-F_{d}(-i, \hat{x}, x)\right],
\end{aligned}
$$

this approximation is valid under the condition that $h(\hat{x})$ is Lipschitz continuous, as $F_{d}$ is given by

$F_{d}=\left\{\begin{array}{r}F\left(\tau_{i} c_{\delta} \delta+\hat{x}-x\right)-F\left(\tau_{i-1} c_{\delta} \delta+\hat{x}-x\right) \\ \text { if } i \in\left\{1, \cdots, \frac{N_{I}}{2}\right\}, \\ F\left(\tau_{i+1} c_{\delta} \delta+\hat{x}-x\right)-F\left(\tau_{i} c_{\delta} \delta+\hat{x}-x\right) \\ \text { if } i \in\left\{-1, \cdots,-\frac{N_{I}}{2}\right\},\end{array}\right.$

the approximation is valid due to the continuity assumptions on $F$.

Estimation bias $\epsilon$ can be obtained through the ODE approximation

$$
\frac{d \epsilon}{d t}=\tilde{h}(\epsilon)
$$

where $\tilde{h}(\epsilon)=h(\epsilon+x)$. Lyapunov theory can be used to show that the algorithm is asymptotically unbiased. Using the quadratic Lyapunov function

$$
\mathcal{L}(\epsilon)=\epsilon^{2},
$$

one needs to show that $\tilde{h}(\epsilon)=0$ for $\epsilon=0$ and $\frac{d \mathcal{L}}{d t}<0$ for $\epsilon \neq 0$. Thus if these conditions are met, the BarbashinKrasovskii theorem [8, Ch. 4] can be used to prove that $\epsilon=0$ is globally asymptotically stable point for (10) and consequently the algorithm is asymptotically unbiased. For doing so, $\tilde{h}(\epsilon)$ can be rewritten as

$$
h(\epsilon)=\sum_{i=1}^{\frac{N_{I}}{2}} \eta_{i}\left[\tilde{F}_{d}(i, \epsilon)-\tilde{F}_{d}(-i, \epsilon)\right]
$$

where $\tilde{F}_{d}(i, \epsilon)=F_{d}(i, \epsilon+x, x)$.

Due to the noise and quantizer symmetry assumptions the condition for $\epsilon=0$ can be easily verified. The condition on 
the derivative

$$
\frac{d \mathcal{L}}{d t}=\frac{d \mathcal{L}}{d \epsilon} \frac{d \epsilon}{d t}=2 \epsilon \tilde{h}(\epsilon)<0, \quad \text { for } \quad \epsilon \neq 0,
$$

can be verified separately for $\epsilon>0$ and $\epsilon<0$ by noting that

- $\eta_{i}$ for $i>0$ are positive by assumption.

- $\tilde{F}_{d}(i, \epsilon)<\tilde{F}_{d}(-i, \epsilon)$ for $\epsilon>0$ due to the assumptions on monotonicity and symmetry of the noise distribution and also to the symmetry of the quantizer.

- $\tilde{F}_{d}(i, \epsilon)>\tilde{F}_{d}(-i, \epsilon)$ for $\epsilon<0$ due to the same assumptions mentioned above.

Therefore, the algorithm is asymptotically unbiased for every combination of $\hat{X}_{0}$ and $x$.

Using also the results in [7, Chap. 4], the minimum asymptotic MSE for the algorithm is

$$
\mathrm{MSE}_{\infty}=\frac{\sigma_{w} \sqrt{2 \boldsymbol{\eta}^{T} \mathbf{F}_{\mathbf{d}} \boldsymbol{\eta}}}{2 \boldsymbol{\eta}^{T} \mathbf{f}_{\mathbf{d}}}
$$

In (14) $\mathbf{F}_{\mathbf{d}}$ is a diagonal matrix $\operatorname{diag}\left[F_{d}[1], \cdots, F_{d}\left[\frac{N_{I}}{2}\right]\right]$, where $F_{d}[i]=F_{d}(i, x, x)$ depends only on $i$. The vector $\mathbf{f}_{\mathbf{d}}$ is $\left[f_{d}[1] \cdots f_{d}\left[\frac{N_{I}}{2}\right]\right]^{T}$, where

$$
f_{d}= \begin{cases}f\left(\tau_{i-1} c_{\delta} \delta\right)-f\left(\tau_{i} c_{\delta} \delta\right), & \text { if } i \in\left\{1, \cdots, \frac{N_{I}}{2}\right\}, \\ f\left(\tau_{i} c_{\delta} \delta\right)-f\left(\tau_{i+1} c_{\delta} \delta\right), & \text { if } i \in\left\{-1, \cdots,-\frac{N_{I}}{2}\right\} .\end{cases}
$$

The optimal $\gamma$ that gives this MSE is

$$
\gamma^{\star}=\frac{\sigma_{w}}{\sqrt{2 \boldsymbol{\eta}^{T} \mathbf{F}_{\mathbf{d}} \boldsymbol{\eta}}} .
$$

Minimization of (14) can be done w.r.t. $\boldsymbol{\eta}$ by solving the following equivalent maximization problem (note that the positive constraint on $\boldsymbol{\eta}$ is neglected)

$$
\arg \max _{\boldsymbol{\eta}}\left\{\frac{\left[\boldsymbol{\eta}^{T} \mathbf{f}_{\mathbf{d}}\right]^{2}}{\boldsymbol{\eta}^{T} \mathbf{F}_{\mathbf{d}} \boldsymbol{\eta}}\right\}
$$

Using the Cauchy-Schwarz inequality or a maximum eigenvalue argument, the optimal $\boldsymbol{\eta}$ can be

$$
\boldsymbol{\eta}^{\star}=\mathbf{F}_{\mathbf{d}}{ }^{-1} \mathbf{f}_{\mathbf{d}}
$$

which is a vector with positive elements $\eta_{i}^{\star}=\frac{f_{d}[i]}{F_{d}[i]}$ that does not depend on $x$. The optimal MSE and $\gamma^{\star}$ are

$$
\operatorname{MSE}_{\infty}=\gamma^{\star}=\sigma_{w}\left(2 \sum_{i=1}^{\frac{N_{I}}{2}} \frac{f_{d}^{2}[i]}{F_{d}[i]}\right)^{-\frac{1}{2}} .
$$

Note that this quantity still depends on $\tau$ and $c_{\delta}$. As the minimization of $\mathrm{MSE}_{\infty}$ can be a very difficult problem for general $\tau$, in the simulation section, uniform quantization will be considered, with the following threshold vector

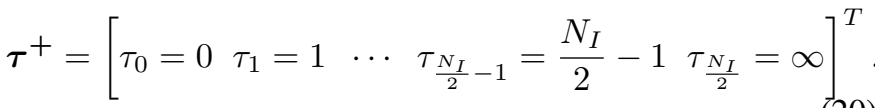

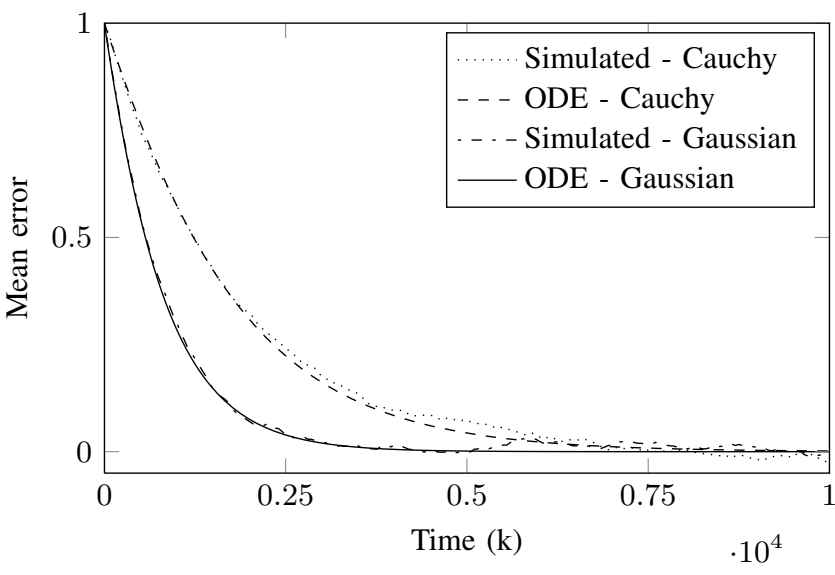

Fig. 2. Mean error for Gaussian and Cauchy noises with $N_{I}=4, \delta=1$, $x=0$ and $\hat{X}_{0}=1$.

\section{Simulation}

The algorithm will be simulated and its performance in terms of mean error and MSE will be compared to the theoretical approximations. The quantization will be considered to be uniform as mentioned above, therefore, the only free parameter for the minimization of $\mathrm{MSE}_{\infty}$ is $c_{\delta}$. This optimization problem can then be solved by grid optimization. After finding the optimal $c_{\delta}$, all the other parameters of the algorithm $\left(\boldsymbol{\eta}, \gamma^{\star}\right)$ can be obtained.

Gaussian and Cauchy distributions will be considered, the former is common choice for measurement noise distribution and the latter is used for simulating the behavior of noise with outliers (heavy tailed noise). Their PDFs for $\delta=1$ are respectively

$$
f_{G}(x)=\frac{1}{\sqrt{\pi}} e^{-x^{2}}, \quad f_{C}(x)=\frac{1}{\pi\left(1+x^{2}\right)} .
$$

For obtaining the simulated mean, Monte Carlo simulation was used. The algorithm was simulated 10 times with 10000 samples for each type of noise distribution and $N_{I}=4$. Other simulation conditions were $x=1, \hat{X}_{0}=0, \delta=1$, $\sigma_{w}=0.001$. The simulated mean error is presented in Fig. 2 with the approximation given by the numerical solution of (10). The simulated results are very close to the theoretical results and the mean error approach zero with increasing number of samples as it was predicted. Also, the algorithm was simulated for $N_{B}=\log _{2}\left(N_{I}\right)=\{1,2,3,4,5\}$ and 10000 realizations with $10^{5}$ samples each to obtain a simulated MSE. The simulated MSE was obtained by calculating the average through the realizations and samples. The scale factors for the noise were $\delta=\sqrt{2}$ for Gaussian noise and $\delta=1$ for Cauchy noise, both with $\sigma_{w}=0.001$. A comparison between the simulated MSE and the MSE in (19) is given in Fig. 3, where the theoretical results are also very close to simulated results.

To evaluate the loss of performance due to quantization, the loss of performance w.r.t. the optimal real-valued measurement estimator was evaluated. In the Gaussian case, the optimal 


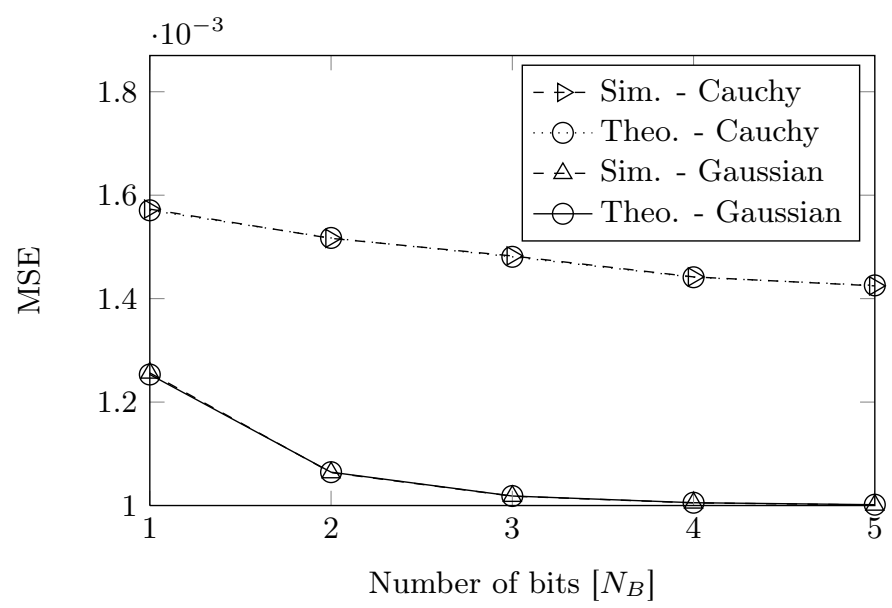

Fig. 3. Simulated and theoretical asymptotic mean squared error (MSE) for Gaussian $(\delta=\sqrt{2})$ and Cauchy $(\delta=1)$ noise with $\sigma_{w}=0.001$ and $N_{B}=\{1,2,3,4,5\}$.

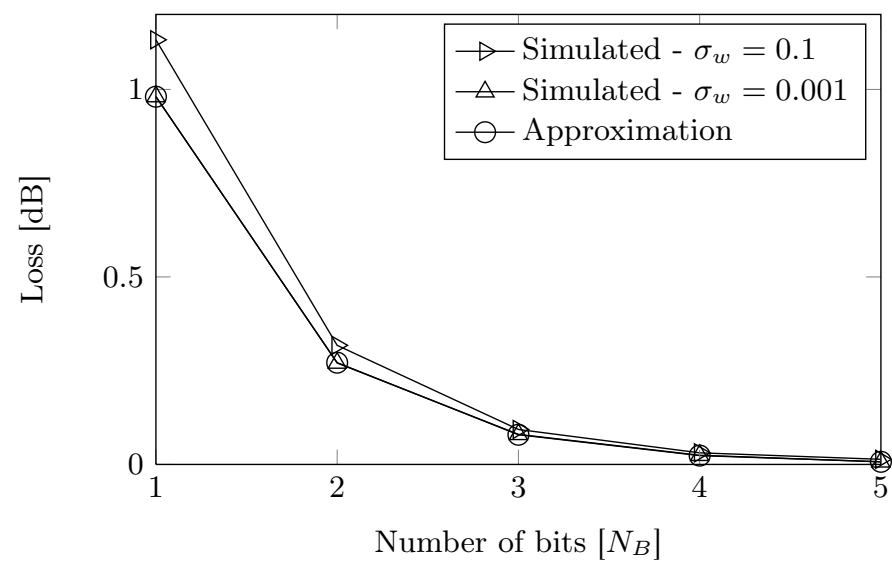

Fig. 4. Simulation and approximation of the loss of performance of the adaptive algorithm with respect to the Kalman filter asymptotic variance for $N_{B}=\{1,2,3,4,5\}, \delta=\sqrt{2}, \sigma_{w}=0.1$ and 0.001 .

estimator based on real-valued measurements is the Kalman filter. Based on the covariance equations of the Kalman filter [9], the asymptotic estimation variance $\mathrm{MSE}_{\infty}^{c}$ is given by

$$
\operatorname{MSE}_{\infty}^{c}=\frac{\sqrt{\sigma_{w}^{4}+2 \delta^{2} \sigma_{w}^{2}}-\sigma_{w}^{2}}{2}
$$

The loss of performance due to quantization is then evaluated in $(\mathrm{dB})$ as

$$
\begin{aligned}
L=10 \log _{10}\left(\frac{\mathrm{MSE}_{\infty}}{\mathrm{MSE}_{\infty}^{c}}\right) & \approx 10 \log _{10}\left(\operatorname{MSE}_{\infty} \sqrt{\frac{2}{\sigma_{w}^{2} \delta^{2}}}\right) \\
& \approx-5 \log _{10}\left(\delta^{2} \sum_{i=1}^{\frac{N_{I}}{2}} \frac{f_{d}^{2}[i]}{F_{d}[i]}\right),
\end{aligned}
$$

where the approximation is valid for small $\sigma_{w}$. The approximation above is shown in Fig. 4 with the simulated loss for $\delta=\sqrt{2}, \sigma_{w}=0.001$ and $\sigma_{w}=0.1$ under the same conditions as for the results in Fig. 3 .

The approximation is good for $\sigma_{w}=0.001$ and it gives close results even for $\sigma_{w}=0.1$. One can also observe that the loss due to quantization has small values for $N_{B}=2$ becoming negligible for $N_{B}=4$. It is interesting to note that the loss for $N_{B}=2$ is far lower than the loss for estimating a constant with $N_{B}=1$ presented in [3], [4], which is $10 \log _{10}\left(\frac{\pi}{2}\right) \approx 1.96 \mathrm{~dB}$. Note that if $N_{B}=1$ is considered for both cases, the loss for tracking is half ( $L \approx 0.98 \mathrm{~dB}$ ) the loss for estimating a constant, indicating that even under minimum quantizer resolution, the performance degradation for tracking is not large.

\section{Conclusions}

In this paper, an adaptive algorithm was proposed for the tracking of a Wiener process based on quantized measurements. The algorithm was shown to be asymptotically unbiased and the optimal parameters of the algorithm were obtained by minimizing the asymptotic MSE. The results both in terms of mean error and MSE were validated by simulation. An interesting observation is that the algorithm based on quantized observations has a negligible loss of performance w.r.t. the optimal real-valued measurement estimator for only a few measurement bits $\left(N_{B}=4\right.$ or 5$)$ and this result holds even for moderate speed of parameter variation. This means that in the bandwidth constrained case, sensor networks with low resolution sensors might be more efficient than single sensor high resolution approaches.

\section{ACKNOWLEDGMENT}

The authors would like to thank the Erasmus Mundus EBWII program for funding this study.

\section{REFERENCES}

[1] D. Puccinelli and M. Haenggi, "Wireless sensor networks: applications and challenges of ubiquitous sensing," Circuits and Systems Magazine, IEEE, vol. 5, no. 3, pp. 19-31, 2005.

[2] A. Gersho and R. Gray, Vector quantization and signal compression. Springer, 1992.

[3] H. Papadopoulos, G. Wornell, and A. Oppenheim, "Sequential signal encoding from noisy measurements using quantizers with dynamic bias control," IEEE Trans. Inf. Theory, vol. 47, no. 3, pp. 978-1002, 2001.

[4] A. Ribeiro and G. Giannakis, "Bandwidth-constrained distributed estimation for wireless sensor networks-part I: Gaussian case," IEEE Trans. Signal Process., vol. 54, no. 3, pp. 1131-1143, 2006.

[5] H. Li and J. Fang, "Distributed adaptive quantization and estimation for wireless sensor networks," IEEE Signal Process. Lett., vol. 14, no. 10, pp. 669-672, 2007.

[6] J. Fang and H. Li, "Distributed adaptive quantization for wireless sensor networks: From delta modulation to maximum likelihood," IEEE Trans. Signal Process., vol. 56, no. 10, pp. 5246-5257, 2008.

[7] A. Benveniste, M. Métivier, and P. Priouret, Adaptive algorithms and stochastic approximations. Springer-Verlag New York, Inc., 1990.

[8] H. Khalil and J. Grizzle, Nonlinear systems. Macmillan Publishing Company New York, 1992.

[9] B. Anderson and J. Moore, Optimal filtering. Prentice-hall Englewood Cliffs, NJ, 1979 